Type of article: Regular article

\title{
Secretion of the housekeeping protein glyceraldehyde-3-phosphate dehydrogenase by the LEE-encoded type III secretion system in enteropathogenic Escherichia coli
}

\author{
Laura Aguilera ${ }^{a}$, Elaine Ferreira ${ }^{a}$, Rosa Giménez ${ }^{a}$, Francisco José Fernández ${ }^{\text {, }}$, Marta \\ Taulés $^{c}$, Juan Aguilar ${ }^{a}$, M. Cristina Vegab ${ }^{b}$,osefa Badia ${ }^{a}$ and Laura Baldomà ${ }^{a, *}$ \\ a Departament de Bioquímica i Biologia Molecular, Institut de Biomedicina de la \\ Universitat de Barcelona (IBUB), Facultat de Farmàcia, Universitat de Barcelona, 08028 \\ Barcelona, Spain \\ b Structural and Quantitative Biology Department, Centro de Investigaciones Biológicas \\ (CIB-CSIC), Ramiro de Maeztu 9, 28040 Madrid, Spain \\ ${ }^{\mathbf{c}}$ Scientific and Technological Centres, Universitat de Barcelona, Baldiri Reixac, 10-12, \\ 08028 Barcelona, Spain \\ * Corresponding author: Departament de Bioquímica i Biología Molecular, Facultat de \\ Farmàcia, Universitat de Barcelona, Av. Diagonal, 643, E-08028-Barcelona, Spain. \\ Phone: 34-934034496. Fax: 34-934024520. E-mail: lbaldoma@ub.edu
}


${ }^{1}$ The abbreviations used are: BSA, bovine serum albumin; BLI, biolayer interferometry; EHEC, enterohemorrhagic Escherichia coli; EPEC, enteropathogenic Escherichia coli; DMEM, Dulbecco's modified Eagle's medium; EDC, N-(3-Dimethylaminopropyl)-N'ethylcarbodiimide; EDTA, ethylenediaminetetracetic acid; GAPDH, glyceraldehyde-3phosphate dehydrogenase; GST, glutathione-S-transferase; IPTG, isopropyl- $\beta$ thiogalactoside; LB, Luria-Bertani broth; LEE, locus of enterocyte effacement; MES, 2-(Nmorpholino)ethanesulfonic acid; NHS, N-hydroxysuccinimide ester; NTA, nitrilotriacetic acid; PVDF, polyvinylidene difluoride; SDS-PAGE, sodium dodecyl sulphatepolyacrylamide gel electrophoresis; TCA, trichloroacetic acid; T3SS, type III secretion system. 


\begin{abstract}
Glyceraldehyde-3-phosphate dehydrogenase (GAPDH) is a multifunctional housekeeping protein secreted by pathogens and involved in adhesion and/or virulence. Previously we reported that enterohemorragic (EHEC) and enteropathogenic (EPEC) Escherichia coli secrete GAPDH into the culture medium. This bacterial protein binds human plasminogen and fibrinogen and remains associated with Caco-2 cells upon infection. In these pathogens, GAPDH secretion is not linked to outer membrane vesicles and depends on growth conditions, although the secretion mechanism is still unknown.
\end{abstract} EPEC is an attaching and effacing pathogen able to secrete and translocate multiple effector proteins into infected cells through a type III secretion system (T3SS). The secretion process is often dependent on a bacterial chaperone. The chaperone CesT displays broad substrate specificity and plays a central role in the recruitment of multiple type III effectors to the T3SS apparatus. Here we provide genetic evidences on GAPDH secretion through T3SS by EPEC grown in DMEM. Secretion of GAPDH is increased in $\triangle s e p D$ mutants and abolished in mutants defective in the type III ATPase EscN. Complementation with escN gene restores GAPDH secretion. In addition, we prove by means of pull down experiments, overlay immunoblotting and biolayer interferometry a novel interaction between GAPDH and the chaperone CesT. This interaction, which is strong and slow dissociating, may stabilise a population of GAPDH molecules in a secretion competent-state and target them to the type III secretion apparatus. This is the first description of CesT interaction with a housekeeping protein and its export through T3SS.

Key words: glyceraldehyde-3-phosphate dehydrogenase, protein secretion, T3SS effector, chaperone CesT, Escherichia coli 


\section{Introduction}

Glyceraldehyde-3-phosphate dehydrogenase (GAPDH, EC 1.2.1.12), a key enzyme in the glycolytic pathway, is considered a multifunctional protein involved in numerous cellular processes in mammalian cells (reviewed in Sirover, 2005, 2011). In bacteria, nonglycolytic GAPDH functions have been associated with its extracellular location. GAPDH is one of the housekeeping proteins secreted by pathogens that interact with different host components, being involved in adhesion and/or virulence (Alvarez-Dominguez et al., 2008; Jin et al., 2005; Lama et al., 2009; Pancholi and Chhatwal, 2003; Schaumburg et al., 2004; Seifert et al., 2003; Terao et al., 2006).

In gram-negative pathogens, GAPDH was shown to be secreted into the medium in a soluble and active form by enterohemorragic Escherichia coli (EHEC) and enteropathogenic E. coli (EPEC). In these pathogens, secretion is not linked to outer membrane vesicles and depends on growth conditions. The protein is secreted at $37^{\circ} \mathrm{C}$ by cells grown in LB or in eukaryotic culture media such as DMEM or Ham's F-12, but not in glucose-minimal medium (Egea et al., 2007). E. coli GAPDH binds human plasminogen and fibrinogen and remains associated with Caco-2 cells upon infection (Egea et al., 2007). In addition, we have shown that the secreted protein can act as a target of oxidation and may therefore protect bacteria against host oxidative response (Aguilera et al., 2009).

Although descriptions of GAPDH on the surface of gram-positive bacteria or yeast can be traced back more than 15 years, the mechanism that directs its secretion is still unknown. In gram-negative bacteria eight major protein secretion systems have been described so far and named from T1SS to T8SS (Desvaux et al., 2009). The type III secretion system (T3SS) is a key determinant of virulence in many gram-negative bacteria, including animal and plant pathogens. T3SS is a complex protein export apparatus 
composed of a multi-ring base, an inner rod and a protruding needle, which facilitates direct delivery of bacterial virulence proteins (effectors) into host cells (Izoré et al., 2011; Marlovits et al., 2006). T3SS effectors play important roles in infection by modulating or disrupting cellular structures and functions or host immune responses (reviewed by Dean and Kenny, 2009).

EPEC and EHEC are members of a closely related family that intimately attach to intestinal epithelial cells and induce characteristic attaching and effacing (A/E) lesions on the host cell, causing diarrhea (Dean et al., 2005; Kaper et al., 2004). The A/E phenotype consists of the loss of microvilli and the accumulation of actin filaments and other cytoskeletal proteins beneath adherent bacteria. In EPEC, the ability to induce the A/E effects is encoded in a 41-gene pathogenicity island, called the locus of enterocyte effacement (LEE) (MacDaniel and Kaper, 1997). The components of the type III secretion system are encoded in this locus together with associated transcriptional regulators, chaperones and effector proteins.

A large number of proteins are secreted by T3SS into culture media (Deng et al., 2010). These protein substrates can be divided in several categories based on their secretion hierarchy. Early substrates are components of the needle and inner rod. The needle complex secretes the LEE-encoded translocators EspA, EspB and EspD (intermediate substrates) before the effectors (late substrates). Translocators are needed for translocation of the effectors into host cells (Luo and Donnenberg, 2006). To date multiple type III effectors have been identified in EPEC, some of them are encoded within the LEE whereas others are encoded in distinct pathogenicity islands through the chromosome (reviewed by Dean and Kenny, 2009). The secretion hierarchy of translocators and effectors is regulated by SepL and SepD (Deng et al., 2005; O'Connell et al., 2004). 
Type III effectors from many pathogens usually have an associated chaperone that is not secreted (class I chaperones) (Izoré et al., 2011; Parsot et al., 2003). These chaperones are generally small soluble proteins $(15-20 \mathrm{kDa})$ with an acidic pI that form homodimers that bind to the amino-terminal region of effectors and remain in the bacterial cell after translocation of the effectors into the host cell. However, in many cases, chaperones are not absolutely required for effector secretion (Parsot et al., 2003). Chaperone-effector interactions occur mainly between secondary structured elements of the effector and hydrophobic patches present on the chaperone dimer (Parsot et al., 2003). Multiples roles have been attributed to these chaperones such as substrate stabilization, prevention of substrate premature interactions with other partners in the bacterial cytoplasm or regulation of virulence gene expression (Page and Parsot, 2002).

In EPEC, the LEE-encoded chaperone CesT was shown to interact with several type III effectors encoded within and outside the LEE pathogenicity island (Thomas et al., 2005; Thomas et al., 2007). Through its interaction with the type III ATPase EscN, CesT promotes the recruitment of the cognate effectors to the T3SS apparatus for efficient secretion (Thomas et al., 2005).

Here we report that T3SS mediates GAPDH secretion in EPEC cells grown in DMEM and provide experimental evidence on a novel interaction between GAPDH and the general T3SS chaperone CesT. This interaction may stabilise a small population of GAPDH molecules in a secretion competent-state and target them to the type III secretion apparatus. This is the first description on CesT interaction with a housekeeping protein and its export through T3SS. 


\section{Materials and Methods}

\subsection{Bacterial strains and growth conditions.}

E.coli strains and plasmids used in this study are listed in Table 1. Bacterial cells were routinely grown at $37^{\circ} \mathrm{C}$ in Luria-Bertani broth (LB). Where indicated cells were grown without shaking at $37^{\circ} \mathrm{C}$ in LB or Dulbecco's modified Eagle's medium (DMEM) in a $\mathrm{CO}_{2}$ incubator. Growth was monitored by measuring the optical density at $600 \mathrm{~nm}\left(\mathrm{OD}_{600}\right)$. When indicated, $5 \mathrm{mM}$ IPTG was added to the medium as inducer of chromosome encoded-lacZ expression. When required, ampicillin $(100 \mu \mathrm{g} / \mathrm{ml})$ or kanamycin $(50 \mu \mathrm{g} / \mathrm{ml})$ was added to the medium.

\subsection{Protein secretion assays.}

Isolation of secreted proteins was performed as previously described (Egea et al., 2007). Briefly, overnight cultures in LB were diluted 1:50 in the indicated culture media and incubated without shaking at $37^{\circ} \mathrm{C}$ in a $5 \% \mathrm{CO}_{2}$ atmosphere. After $6-8 \mathrm{~h}$ of growth $\left(\mathrm{OD}_{600}\right.$ of 0.8$)$ bacteria were collected by centrifugation $\left(5,000 \times \mathrm{g}, 10 \mathrm{~min}, 4^{\circ} \mathrm{C}\right)$, and the supernatant was passed through a $0.22 \mu \mathrm{m}$-pore-size filter (Millipore). The proteins in the filtrate were precipitated by incubation on ice for at least $1 \mathrm{~h}$ with $10 \%$ trichloroacetic acid (TCA). When indicated, $0.1 \%$ bovine serum albumin (BSA) was added to the filtered supernatant before TCA precipitation. The protein pellet was washed in $90 \%(\mathrm{v} / \mathrm{v})$ ice-cold acetone, air-dried and suspended in loading buffer before being resolved by SDS-PAGE (Laemmli, 1970). Collected bacteria were resuspended in loading buffer and kept for SDS-

PAGE analysis. 


\subsection{DNA manipulation.}

Bacterial genomic DNA was obtained using the Wizard Genomic DNA purification kit (Promega), and plasmid DNA was prepared using the Wizard Plus SV Midipreps DNA purification system (Promega). DNA manipulations in vitro were performed according to standard protocols (Sambrook and Rusell, 2001). DNA fragments were amplified by PCR using chromosomal DNA as template. When necessary, specific restriction sites were incorporated at the 5'-end of the primers to facilitate the cloning of the fragments in the appropriate vector. PCR reactions were performed with $P f u$ DNA polymerase in standard conditions. DNA was sequenced using an automated ABI 377 DNA sequencer and fluorescent dye termination methods.

To obtain a plasmid construct expressing $\mathrm{His}_{6}-\mathrm{CesT}$, the $\operatorname{ces} T$ gene was amplified by PCR using EPEC E2348/69 genomic DNA and primers CesT-pQE30-bam.fw (5' -CGCGGATCCATGTCATCAAGATCTGAAC-3') and CesT-pQE30-hind.rv (5' -CGCAAGCTTTTATCTTCCGGCGTAATAATG-3') and cloned into BamHI/HindIII digested plasmid pQE30 (Qiagen), yielding pQE-CesT. Restriction sites incorporated at the 5'end of the primers are underlined. The forward primer was designed to fuse the His 6 -tag in frame with cesT. Plasmid DNA was sequenced to ensure that no mutations have been introduced during the amplification reaction.

\subsection{Pull-down experiments with His $\sigma_{-}$CesT.}

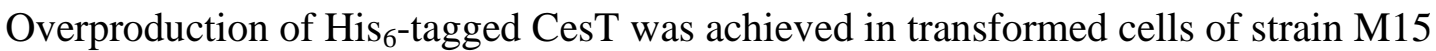
[pREP4] bearing the recombinant plasmid pQE-CesT after isopropyl- $\beta$-thiogalactoside (IPTG) induction $(0.3 \mathrm{mM})$ in LB-ampicillin-kanamycin medium for $16 \mathrm{~h}$ at $37{ }^{\circ} \mathrm{C}$. The 
protein was purifed under native conditions with $\mathrm{Ni}^{2+}$-nitrilotriacetic acid (NTA) resin (Qiagen). Centrifugations were performed at $4{ }^{\circ} \mathrm{C}$, and column chromatography was carried out at room temperature. The cell pellet from a $15 \mathrm{ml}$ culture of strain M15 [pREP4] bearing plasmid pQE-CesT was suspended in $1 \mathrm{ml}$ of $50 \mathrm{mM}$ sodium phosphate buffer, $\mathrm{pH}$ 8.0, containing $10 \mathrm{mM}$ imidazole and $300 \mathrm{mM} \mathrm{NaCl}$ (binding buffer), and sonicated on ice. The cell lysate was centrifuged at $15000 \mathrm{~g}$, and the supernatant was incubated with $0.1 \mathrm{ml}$ of $\mathrm{Ni}^{2+}$-NTA resin for $1 \mathrm{~h}$ at $4^{\circ} \mathrm{C}$ with gentle shaking. After loading the mixture into a column, the resin was washed with $50 \mathrm{mM}$ sodium phosphate buffer, $\mathrm{pH} 8.0$, containing 20 $\mathrm{mM}$ imidazole and $300 \mathrm{mM} \mathrm{NaCl}$ (wash buffer).

Column-bound $\mathrm{His}_{6}$-CesT was equilibrated with binding buffer, followed by the addition of $0.5 \mathrm{ml}$ of an EPEC cell extract $(10 \mathrm{mg} / \mathrm{ml})$ in binding buffer obtained from cells grown in DMEM by sonic disruption as described above. The flow-through fraction was kept for analysis. The column was washed 4 x $5 \mathrm{ml}$ of wash buffer. A last $0.1 \mathrm{ml}$ wash was performed and kept for gel analysis. Recombinant $\mathrm{His}_{6}-\mathrm{CesT}$ and interacting proteins were then eluted with $0.1 \mathrm{ml}$ of elution buffer $(50 \mathrm{mM}$ sodium phosphate buffer, $\mathrm{pH}$ 8.0, containing $300 \mathrm{mM}$ imidazole and $300 \mathrm{mM} \mathrm{NaCl}$ ) followed by SDS-PAGE and immunoblotting analysis.

\subsection{Immunoblotting analysis.}

For Western blot analysis, protein samples were separated on 10\% SDS-PAGE and transferred to a HyBond-P polyvinylidene difluoride (PVDF) membrane by using a BioRad MiniTransblot apparatus. Equal numbers of bacteria were used to prepare samples (whole cell lysates or secreted proteins) for analysis. The membrane was blocked in PBS0.05\% Tween-20 and 5\% skimmed milk (blocking solution) for $1 \mathrm{~h}$ at room temperature, 
and then incubated with specific antibodies against GAPDH (1:5,000 dilution in blocking solution) (Egea et al., 2007) or with antibodies against $\beta$-galactosidase (Abcam, 1:10,000 dilution) for $16 \mathrm{~h}$ at $4^{\circ} \mathrm{C}$. The secondary antibody was donkey anti-rabbit immunoglobulin horseradish peroxidase-linked, diluted 1:15,000 in blocking solution. Incubation with the secondary antibody was omitted when anti-His horseradish peroxidase-linked antibody (GenScript; 1:5,000 dilution) was used as primary antibody. The protein-antibody complex was visualized by using the ECL Plus Western blotting detection system (Amersham Pharmacia Biotech). Control gels were stained with Sypro® Ruby or Coomassie Brilliant Blue R-250.

Binding of GAPDH to CesT was analyzed by Far-Western assays (overlay immunoblotting). For these experiments recombinant GAPDH was expressed and purified using the Glutathione-S-transferase (GST) gene fusion system with recognition sites for factor Xa cleavage as described in detail previously (Egea et al., 2007). Purified GAPDH was further purified by affinity chromatography with anti-factor Xa-agarose resin (Sigma) to remove factor Xa.

For mono-dimensional Far-Western experiments, purified GAPDH $(2.5 \mu \mathrm{g})$ was separated on $10 \%$ SDS-PAGE and transferred to a PVDF membrane. After being blocked overnight with $1 \%$ gelatin in TBS buffer $(20 \mathrm{mM}$ Tris- $\mathrm{HCl}, 150 \mathrm{mM} \mathrm{NaCl}, \mathrm{pH} 7.6)$, the membrane was incubated with purified $\mathrm{His}_{6}$-CesT $(1$ or $5 \mu \mathrm{g} / \mathrm{ml})$ diluted in TBS- $0.05 \%$ Tween-20 for 3 hours and then washed four times in the same buffer. To visualize interaction of GAPDH with CesT, the membrane was incubated with anti-His specific antibodies (1:5,000 dilution TBS-1\% gelatin-0.05\% Tween-20) for $16 \mathrm{~h}$ at $4^{\circ} \mathrm{C}$, and processed as described above using the ECL Western blotting kit to visualize the reactive 
spots. The incubation step with purified $\mathrm{His}_{6}$-CesT was omitted as negative control. When indicated, purified GAPDH (5 $\mu \mathrm{g})$ was subjected to two-dimensional gel electrophoresis before being blotted onto the PVDF membrane, which was then incubated with purified His $_{6}$-CesT $(1 \mu \mathrm{g} / \mathrm{ml})$ and processed as described above.

\subsection{Two-dimensional gel electrophoresis.}

2D gel electrophoresis was performed using the Protean IEF-Cell (Bio-Rad). Purified recombinant GAPDH (5 $\mu \mathrm{g})$ was diluted in $125 \mu \mathrm{l}$ of rehydration buffer $(9 \mathrm{M}$ urea, $4 \%$ CHAPS, $50 \mathrm{mM}$ dithiothreitol, $0.5 \%$ immobilized $\mathrm{pH}$ gradient buffer and traces of bromophenol blue). Isoelectric focusing was performed in immobilized $\mathrm{pH}$ 5-8 gradient strips (BioRad). Second dimension SDS-PAGE was performed on $12.5 \%$ acrylamide gels that were processed for immunoblotting analysis. Parallel gels were silver-stained to visualize total protein.

\subsection{Biolayer interferometry.}

Octet Red (Fortebio) instrument with amine reactive biosensors was used to study kinetics of GAPDH - CesT interaction. Carboxylic groups on the sensor surface were activated by incubation with $0.2 \mathrm{M}$ [N-(3-Dimethylaminopropyl)-N'-ethylcarbodiimide] (EDC):0.05 M [N-hydroxysuccinimide ester] (NHS) during $10 \mathrm{~min}$. The ligand CesT diluted in MES buffer $\mathrm{pH} 4.0$ (final concentration of $50 \mu \mathrm{g} / \mathrm{ml}$ ) was then loaded on activated biosensors (10 min incubation). The unreacted NHS-esters were quenched with $0.05 \mathrm{M}$ ethanolamine for $10 \mathrm{~min}$. All experiments were conducted at $30^{\circ} \mathrm{C}$ with agitation set to $1000 \mathrm{rpm}$ in $10 \mathrm{mM}$ HEPES pH 7.5, $150 \mathrm{mM} \mathrm{NaCl,} 3 \mathrm{mM}$ EDTA, 0.01\% (w/v) Tween- 
20 and $0.1 \mathrm{mg} / \mathrm{ml}$ BSA. Biosensors with immobilized CesT were exposed to different concentrations of purified GAPDH $(0-5.5 \mu \mathrm{M})$. After dipping the biosensor with each concentration of the analyte GAPDH, the surface was regenerated with $0.1 \%$ SDS during

30 seconds. This allowed it to return at the baseline. Data analysis and curve fitting were done using the ForteBio Data Analysis software version 6.3 provided by the manufacturer. Kinetic constants were obtained by fitting data to a 1:1 model after parallel reference sensor subtraction.

\section{Results and discussion}

\subsection{GAPDH secretion through the LEE-encoded T3SS in EPEC.}

Secretion of GAPDH by EPEC in culture media known to induce the expression of LEE-encoded genes (Egea et al., 2007) prompted us to examine whether the LEE-encoded T3SS is involved in GAPDH secretion in this pathogen. To this end, GAPDH secretion was analyzed in $e s c N$ or $e s p B$ mutant strains. It is well known that mutations in $e s c N$, the gene encoding the T3SS ATPase, abolish secretion of both translocator and effector proteins through this system. In contrast, mutations in $\operatorname{esp} B$, the gene encoding a hydrophobic translocon protein that is thought to form with EspD a translocation pore through the host membrane, disrupt translocation of effector proteins into host cells during infection but do not impair secretion of these proteins into the extracellular medium (Deng et al., 2004, Luo and Donnenberg, 2006, Luo and Donenberg, 2011).

Cells of wild-type EPEC E2348/69 and the derived mutant strains CVD452 $(e s c N:: a p h A 3)$ and UMD864 $(\Delta e s p B)$ were grown statically at $37^{\circ} \mathrm{C}$ in DMEM or LB (a medium that promotes down-regulation of LEE-encoded T3SS) to an $\mathrm{OD}_{600}$ of 0.8 . When 
indicated, cultures were incubated overnight. Proteins in the cell-free culture supernatants were collected by TCA precipitation and the presence of secreted GAPDH in these fractions was analyzed by Western blot. To rule out cytosolic contamination due to cell lysis, immunoblots were revealed with antibodies against the intracellular protein $\beta$ galactosidase (Fig. 1). Expression of $\beta$-galactosidase was achieved by addition of $5 \mathrm{mM}$ IPTG to the culture medium. Gels run in parallel were stained with Sypro® Ruby to visualize total secreted protein.

In cells grown in LB, no significant changes in the secretion profiles of the three strains were observed (Fig. 1A). In these growth conditions GAPDH was secreted by all strains at the same extent. The absence of $\beta$-galactosidase in culture supernatants ruled out cell lysis phenomena, and indeed proves GAPDH secretion by LB-grown EPEC through a non-dependent T3SS mechanism.

Regarding DMEM-cultured cells, as expected from the mutant phenotypes, the secretion profiles of wild-type EPEC and $\triangle \operatorname{esp} B$ mutant were very similar, except for the 34 $\mathrm{kDa}$-protein band (EspB) that is absent from the UMD864 cell-free supernatant. The secretion profile was clearly different for the escN mutant. In this case, almost no secreted proteins were apparent in cell-free supernatants (Fig. 1A). The espB mutant UMD864 displayed reduced GAPDH secretion in DMEM compared with the wild-type strain. In this mutant, GAPDH was immunodetected in supernatants of overnight DMEM cultures (Fig. 1A). In contrast, secretion of GAPDH was completely abolished in the escN insertion mutant CVD452 grown in DMEM at any incubation time (Fig. 1A). The lack of GAPDH secretion in EPEC cells defective in the T3SS ATPase was confirmed with a non-polar $\Delta e s c N$ mutant (Gauthier and Finlay, 2003) (Fig. 1B). To normalize protein precipitation 
efficiency, TCA precipitation of cell free-supernatants was also performed in the presence of $0.1 \%$ BSA as a carrier. Immunoblotting analysis also failed to detect secreted GAPDH in both $\operatorname{escN}$ mutants in these conditions (not shown). GAPDH secretion was restored in the escN mutants by plasmid pCVD446 bearing the wild-type escN gene (Fig. 1B). Overall, these results indicate that GAPDH may be secreted by the LEE-encoded T3SS in EPEC grown in DMEM.

In EPEC cells defective in SepD function, the secretion of type III translocon components is reduced while the T3SS-dependent secretion of several effectors is highly increased, hence aiding the identification of previously unrecognized type III secreted proteins (Deng et al., 2004, 2005, O'Connell et al., 2004). Therefore, we set out to evaluate GAPDH secretion in DMEM cultures of a $\triangle \operatorname{sep} D$ mutant strain (Deng et al., 2005). Equal numbers of bacteria were used to obtain the secreted proteins for Western blot analysis. As shown in Fig.1C, GAPDH secretion was enhanced in the $\triangle s e p D$ mutant when compared with the wild-type strain.

Results obtained with the espB, escN and $\operatorname{sep} D$ mutants show that GAPDH secretion profile follows that of the bona fide T3SS effectors thereby pointing to GAPDH as a putative type III effector protein in EPEC.

\subsection{Interaction of GAPDH with the general type III chaperone CesT.}

Although CesT was first described as a specific chaperone for the type III effectors Tir and Map, Thomas et al. $(2005,2007)$ showed, through an elegant work, that CesT is required for efficient secretion of many other type III effectors, some of them not encoded in the LEE pathogenicity island. We therefore set out to evaluate whether CesT can bind 
GAPDH. To this end, His-tagged CesT was purified and bound to a $\mathrm{Ni}^{2+}$-NTA column followed by the addition of wild-type EPEC cell extract $(0.5 \mathrm{ml} ; 10 \mathrm{mg} / \mathrm{ml})$ in binding buffer obtained from cultures grown in DMEM. In this experiment, proteins that interact with CesT are retained on the column and then can be co-eluted with CesT. After extensive washing, His ${ }_{6}$-CesT was eluted with elution buffer. The purification fractions were then subjected to SDS-PAGE followed by Western blot analysis with antibodies against GAPDH. Parallel samples were revealed by Coomassie blue staining. As shown in Fig. 2 the final wash fraction did not contain any detectable protein. In the eluted fraction, in addition to the dominant protein $\mathrm{His}_{6}$-CesT, other proteins were visible. These proteins may correspond to EPEC effectors interacting with CesT (Thomas et al., 2005). Immunoblotting of the column fractions with anti-GAPDH antibodies indicated that a fraction of this protein was bound and co-eluted with $\mathrm{His}_{6}$-CesT (Fig. 2, left panel). In a control experiment, the EPEC cell extract was directly applied to the $\mathrm{Ni}^{2+}$-NTA column and processed in parallel. Immunoblotting of the eluted fraction showed that the $\mathrm{Ni}^{2+}-\mathrm{NTA}$ resin $\operatorname{did}$ not bind GAPDH to any extent (Fig. 2, right panel).

Since some type III efectors such as Tir directly bind EscN (Gauthier and Finley, 2003), and this ATPase binds CesT, we set out to examine whether the observed GAPDHCesT interaction was dependent on $\mathrm{EscN}$. Thus, the CesT column binding experiment was also performed with $\triangle e s c N$ cell extracts obtained from DMEM cultures (Fig. 2, middle panel). Immunoblotting of the eluted fractions with anti-GAPDH antibodies revealed that GAPDH was bound on the column and co-eluted with $\mathrm{His}_{6}-\mathrm{CesT}$, thus indicating that EscN is not necessary for CesT and GAPDH interaction. Retention of GAPDH on the CesTaffinity column was also observed with cell extracts of the laboratory strain E.coli 
M15[pREP4]. This result indicates that GAPDH interaction with CesT cannot be attributed to other bridging T3SS proteins and suggests direct GAPDH-CesT interaction.

Direct binding of GAPDH to CesT was confirmed by mono-dimensional Far-Western assays in which purified GAPDH was subjected to SDS-PAGE, transferred to PVDF membrane and reacted with soluble purified His ${ }_{6}$-CesT (Fig. 3A). Since GAPDH is a target of multiple post-translational modifications we set out to analyze whether CesT binding activity is specifically linked to one of these forms. This was approached by Far-Western experiments, in which purified GAPDH was subjected to two-dimensional gel electrophoresis. Purified GAPDH yielded several spots that interacted with CesT (Fig. 3B), thus providing evidence that this binding is not strictly dependent on a GAPDH form.

The dissociation constants of GAPDH-CesT interaction were measured by biolayer interferometry (BLI), a technique that quantitates molecular interactions and provides the on/off rates. When we immobilized CesT and measured the affinity of the GAPDH-CesT complex (Fig. S1, supplemental material), we obtained a $K_{\mathrm{D}}$ of $560 \mathrm{nM}$ and a dissociation rate ( $k$ off) of the complex of $0.0015 \mathrm{~s}^{-1}$. Saturation kinetics with GAPDH could not be observed because only a minor fraction of GAPDH, presumably monomeric, was competent for CesT binding. The reverse experiment could not be performed because immobilization of GAPDH using amine chemistry led to the inactivation of GAPDH-CesT binding motifs.

Even though the structural basis for GAPDH-CesT interaction is not yet known (nor is there a structure of CesT in complex with any of its effector proteins), the requirements for class I chaperone binding are likely to play important roles in shaping the interaction with GAPDH. In particular, the putative degenerate CesT-interacting aminoterminal domain proposed for type III effectors of EPEC (Thomas et al., 2007) spans a 
stretch of 60-120 amino acids. If the GAPDH-CesT interaction surface resembles the extended complexes described for analogous chaperone-effector interactions, such as SicPSptP (35-139) (PDB ID: 1JYO) (Stebbins and Galán, 2001) and SycE-YopE (17-85)

(PDB ID: 1L2W) (Birtalan et al., 2002) (Fig. 4A), the $120 \mathrm{~N}$-terminal amino-acid residues of GAPDH could adopt a similarly extended conformation to bind the four canonical hydrophobic patches exposed across the chaperone dimer. Indeed, the secondary structure and the pattern of conserved hydrophobic and positively charged residues that are thought to mediate the interaction of T3SS effectors and their cognate chaperones are discernable, despite considerable degeneracy, along the GAPDH amino terminus (Fig. 4B). Since the nascent, monomeric chain of GAPDH has been shown to interact with various chaperones and chaperonins as it emerges from the ribosome, like Trigger factor (Liu et al., 2010) and GroEL/GroES (Houry et al., 1999), it becomes plausible for CesT to compete for a small fraction of nascent GAPDH amino termini thereby interfering with its intracellular stabilization and folding, and targeting GAPDH instead for T3SS-mediated secretion, with proper folding and oligomerization taking place after translocation. The engagement of the GAPDH N-terminal segment in the interaction with CesT would imply the partial disruption of the GAPDH tetramer, since residues 41-51 contribute to the stabilization of the oligomerization interface (Fig. 4C). This would be in keeping with the proposal that proteins targeted for secretion via T3SS should translocate in an unfolded, or partially folded, non-globular state through the $25-\AA$-wide inner diameter of the needle complex (Stebbins and Galán, 2003). Taken together, these results indicate that a stable complex exists between the T3SS chaperone CesT and a particular GAPDH conformation, thereby providing a solid experimental underpinning for the redirection of a fraction of cytosolic GAPDH through the T3SS secretion system. 
To analyze whether CesT function is essential for GAPDH secretion, immunoblotting experiments were performed with cell-free supernatants of DMEM cultures of a $\Delta c e s T$ mutant strain (Abe et al., 1999). Equal numbers of bacteria (wild type EPEC and the $\Delta c e s T$ mutant) were used to obtain secreted proteins for analysis. As shown in Fig.1C, GAPDH secretion was not abolished but reduced in the $\Delta c e s T$ mutant. The fact that the cognate chaperone is not absolutely required for protein secretion seems to be rather common for type III effectors in several pathogens (Parsot et al., 2003). Actually, there is evidence that Tir secretion is not strictly dependent on CesT as low levels of Tir can be detected in culture supernatants of cesT mutants (Elliot et al., 1999).

This study describes for the first time the interaction of a housekeeping protein with the type III chaperone CesT. Interaction of GAPDH with this chaperone may provide the way by which GAPDH is targeted to the type III secretion apparatus and reinforce the idea that GAPDH is secreted by the LEE-encoded T3SS in EPEC cells grown in DMEM.

\subsection{A T3SS-independent GAPDH secretion pathway is operative in EPEC and probiotic E.} coli strains grown in $L B$.

Immunodetection of GAPDH in cell-free supernatants of the $\operatorname{esc} N$ mutant strain CVD452 grown in LB (Fig. 1) indicated that a secretion system other than T3SS is responsible for GAPDH secretion in EPEC grown in this rich bacteriological medium. At this point we set out to study whether this alternative secretion system may also be active in non-pathogenic E. coli strains. In fact, in the last years several reports describe GAPDH secretion by some gram-positive probiotic strains of the genus Lactobacillus (Hurmalainen et al., 2007; Sanchez et al., 2008, 2009a, 2009b). Thus, we decided to analyze GAPDH 
secretion in cultures of some gut microbiota E. coli strains: (i) strains isolated from stool of healthy people (EcoR12, EcoR26 and EcoR42) available from the Selander collection (Ochman and Selander, 1984) and (ii) the probiotic E. coli strain Nissle 1917 (serotype O6:K5:H1). These bacterial cells were grown statically at $37^{\circ} \mathrm{C}$ in DMEM or LB to an $\mathrm{OD}_{600}$ of 0.8 . Presence of secreted GAPDH in the culture supernatants was analyzed by Western blot. None of the strains analyzed secreted GAPDH when grown in DMEM (Fig. 5). These strains do not contain in their chromosome the T3SS-encoding genes. Therefore, this result is in accordance with the involvement of the LEE-encoded T3SS in GAPDH secretion in EPEC grown in DMEM. In contrast, great differences in GAPDH secretion among strains were observed in LB cultures. Extracellular GAPDH was detected in the supernatant of the probiotic strain Nisssle 1917, but not in the supernatants of the natural isolates EcoR26 (Fig. 5), EcoR12 and EcoR41 (not shown). These results indicate that the secretion mechanism responsible for GAPDH export in LB is active in both pathogenic and probiotic E. coli strains. It may be hypothesized that GAPDH secretion may confer to these strains an advantage in the context of gut colonization with respect to other components of the intestinal microbiota. In fact, GAPDH secretion and its interaction with extracellular host components such as mucin or extracellular matrix proteins are rather common among diverse pathogens and probiotic microorganisms (Hurmalainen et al., 2007; Kinoshita et al., 2008; Lama et al., 2009; Pancholi and Chhatwal, 2003).

Results presented in this study indeed prove that secretion of GAPDH in EPEC is mediated by at least two alternative pathways depending on the growth conditions: (i) the pathogen specific T3SS in cells grown in DMEM, and (ii) a system not yet identified in cells grown in LB, which is not restricted to pathogens but also operative in probiotic $E$. 
coli strains. The widespread presence of multiple GAPDH secretion pathways is compatible with GAPDH roles in colonization and infection processes.

\section{Acknowledgments}

This study was supported by the Spanish Ministry of Science and Innovation grants BFU 2007-63090 and BFU2010-22260-C02-01 to LB and PET2008_0101, BIO200911184 and BFU2010-22260-C02-02 to MCV and by the EC project ComplexINC (Framework Programme 7 (FP7) under grant agreement no. 279039) to MCV. We thank Michael S. Donnenberg for providing strains CVD452 and UMD864 as well as plasmid pCVD446. We also acknowledge B. Brett Finlay for providing wild-type EPEC E2348/69 streptomycin resistant, and the derived $\Delta s e p D$ and $\Delta c e s T$ mutants. The probiotic Escherichia coli strain Nissle 1917 was provided by Ardeypharm GmbH, Herdecke, Germany. We also thank W. Deng for technical suggestions. 


\section{Figure Legends}

Fig. 1. Immunoblot analysis of GAPDH secretion by T3SS mutant derivatives of EPEC E23448/69. (A) The mutant strains UMD864 ( $\triangle e s p B)$, CVD452 (escN::aphA3) and the parental wild-type strain EPEC E23448/69 were grown statically in LB or DMEM to an $\mathrm{OD}_{600}$ of 0.8 (6-8 hours). When indicated cultures were grown overnight (o/n). IPTG was added at $5 \mathrm{mM}$ concentration to induce lac $Z$ expression. Aliquots of the culture supernatants ( $\mathrm{SN})$ were precipitated with TCA and analyzed by Western blot using antiGAPDH specific antibodies, or anti- $\beta$-galactosidase as a control of cytosolic contamination. Parallel gels were stained with Sypro® Ruby to visualize total secreted protein. An arrowhead next to the molecular mass marker indicates the position of GAPDH (35 kDa). Whole cell lysates (WL) were prepared from the same samples and analyzed with the indicated antibodies. (B) Complementation of $\Delta e s c N$ with plasmid pCVD446 expressing EscN. Cell-free supernatants from exponentially grown DMEM cultures ( $\mathrm{OD}_{600}$ of 0.8$)$ were precipitated with TCA and analyzed by Western blot using anti-GAPDH or anti- $\beta$ galactosidase specific antibodies. (C) GAPDH secretion profile of $\Delta s e p D$ and $\Delta c e s T$ mutants compared with wild-type EPEC. Supernatants were collected from exponentially grown DMEM cultures $\left(\mathrm{OD}_{600}\right.$ of 0.8$)$ and processed as described above. Equal numbers of bacteria were used to prepare samples for analysis.

Fig. 2. Pull-down experiments showing binding of GAPDH to CesT. Cell extracts obtained from wild-type strain EPEC E23448/69 (left panel) or from the escN mutant strain CVD452 (middle panel) grown in DMEM were passed over Ni-NTA column immobilized His ${ }_{6}$-cesT. Column fractions were analyzed either by Western blot with anti-GAPDH antibodies or by 
Coomassie blue staining. An arrowhead next to the molecular mass marker indicates the position of GAPDH (35 kDa). As a control, the EPEC cell extract was directly applied to the Ni-NTA column and processed in parallel (right panel). CE, cell extract; FT, flowtrough; W, final wash; E, eluted fraction.

Fig. 3. Far-Western analysis of binding of soluble CesT to PVDF-immobilized GAPDH. (A) Mono-dimensional Far-Western analysis. Samples containing $2.5 \mu \mathrm{g}$ protein (purified His $_{6}$-CesT or GAPDH) were subjected to $10 \%$ SDS-PAGE, and the gel was either Coomassie blue-stained or electroblotted. The PVDF membrane was reacted with recombinant $\mathrm{His}_{6}$-CesT $(1 \mu \mathrm{g} / \mathrm{ml}$ or $5 \mu \mathrm{g} / \mathrm{ml})$, subsequently incubated with antibodies antiHis horseradish peroxidase-linked antibody and processed to visualize the reactive bands. As a control, the panel labelled as (-) shows the result of this analysis when the incubation step with purified $\mathrm{His}_{6}$-CesT was omitted. (B) Bi-dimemsional Far-Western analysis. Samples containing $5 \mu \mathrm{g}$ of purified GAPDH were subjected to two-dimensional gel electrophoresis and the gel was either silver-stained (first panel) or electroblotted. The PVDF membrane was reacted with purified $\mathrm{His}_{6}$-CesT $(5 \mu \mathrm{g} / \mathrm{ml})$, subsequently incubated with antibodies anti-His and processed to visualize the reactive bands (second panel). The third panel shows the result of this analysis when the incubation step with recombinant CesT was omitted.

Fig. 4. Structural requirements for CesT-GAPDH interaction. (A) Cartoon representation of the YopE-SycE (PDB ID: 1L2W) and SicP-SptT (PDB ID: 1JYO) crystal structures shown in two views related by a $90^{\circ}$ rotation around a horizontal axis. Chaperones (SycE 
and SicP) are coloured in grey surrounded by their semi-transparent molecular surface and effectors (YopE and SptP) in light orange. Side chains in sticks (CPK colours) correspond to amino acid residues relevant for the chaperone-effector interaction; in particular, a conserved glutamic acid residue on the chaperones (E115 and E118) and the effector $\beta$ strands (and preceding conserved arginine/lysine residues) and central $\alpha$-helix (and preceding conserved aspartic acid), are highlighted. (B) Annotated amino terminal sequences of YopE and SptP effector proteins and E. coli GAPDH (GapA) starting at residues 28,47 , and 23 , respectively, with the experimentally determined secondary structure underneath $(\mathrm{H}=\alpha$-helix $; \mathrm{E}=$ extended $\beta$-strand; $\mathrm{B}=$ isolated $\beta$-bridge; $\mathrm{T}=$ hydrogen-bonded turn; $\mathrm{S}=$ bend; $\mathrm{G}=3_{10}$ helix). Important secondary structures are shown in blue ( $\beta$-strands) or red ( $\alpha$-helices) against a grey background. Key conserved residues lying not necessarily in secondary structures are coloured and marked with an asterisk $(\mathrm{R} / \mathrm{K}$, in blue), a plus sign (Y/F, in red), or shown in red (aspartic acid preceding central $\alpha-$ helix in effectors). The number of residues required for fully covering the chaperone's interaction surface depends on the conformation of the effector. When the effector is mostly in an extended conformation (YopE), the region between the first conserved arginine/lysine and the last residue in the last $\beta$-strand ranges from $31-35$ residues. In contrast, the more condensed $\alpha$-helical structure of SptP (with a non-interacting, greyed-down $\alpha$-helical insertion and a third $\alpha$-helix) allows fitting 71 residues over the SicP chaperone. The effector sequence and secondary-structure motifs recognizable in GAPDH are labelled accordingly. (C) GAPDH monomer and dimer structure (PDB ID: 1DC5) (Yun et al., 2000) shown in cartoons and coloured in rainbow colours. Blue asterisks and plus signs have the same meaning as in (A) and (B). N-terminal GAPDH residues predicted to interact with 
CesT are shown in sticks and CPK colours. In particular, the amino acid stretch 41-51 forms part of a dimeric interface involving a patch of C-terminal residues (shown also in sticks) that is framed by dashed lines. The two symmetric interfaces bury a combined 980 $\AA^{2}$ surface area stabilized by two symmetric sets of 6 hydrogen bonds and 1 salt bridge.

Fig. 5. Immunoblot analysis of GAPDH secretion by several non-pathogenic E.coli strains. The indicated strains were grown in DMEM or LB to an $\mathrm{OD}_{600}$ of 0.8 in the presence of 5 $\mathrm{mM}$ IPTG to induce $l a c Z$ expression. Aliquots of the culture supernatants $(\mathrm{SN})$ were precipitated with TCA and analyzed by Western blot. Whole cell lysates (WL) were prepared from the same samples and analyzed in parallel. 


\section{References}

Abe A, Degrade M, Pfuetzner RA, Sanchez-Sanmartín C, Devinney R, Puente JL, et al. Enteropathogenic Escherichia coli translocated intimin receptor, Tir, requires a specific chaperone for stable secretion. Mol Microbiol 1999;33:1162-75.

Alvarez-Dominguez C, Madrazo-Toca F, Fernandez-Prieto L, Vandekerckhove J, Pareja E, Tober R et al. Characterization of a Listeria monocytogenes protein interfering with Rab5a. Traffic 2008;9:325-37.

Aguilera L, Giménez R, Badia J, Aguilar J, Baldoma L. NAD ${ }^{+}$-dependent post-translational modification of Escherichia coli glyceraldehyde-3-phosphate dehydrogenase. Int Microbiol 2009;12:187-92.

Birtalan SC, Phillips RM, Ghosh P. Three-dimensional secretion sugnals in chaperoneeffector complexes of bacterial pathogens. Mol Cell 2002;9:971-980.

Dean P, Maresca M, Kenny B. EPEC's weapons of mass subversion. Curr Opin Microbiol $2005 ; 8: 28-34$.

Dean P, Keny B. The effector repertoire of enteropathogenic E. coli: ganging up on the host cell. Curr Opin Microbiol 2009;12:101-09.

Deng W, Puente JL, Gruenheid S, Li Y, Váyanse BA, Vázquez A, Barba J et al. Dissecting virulence: Systematic and functional analyses of a pathogenicity island. Proc Natl Acad Sci 2004;101:3597-02.

Deng W, Li Y, Hardwidge PR, Frey EA., Pfuetzner RA, Lee S et al. Regulation of type III secretion hierarchy of translocators and effectors in attaching and effacing bacterial pathogens. Infect Immun 2005;73:2135-46. 
Deng W, Hoog CL, Yu HB, Li Y, Croxen MA, Thomas NA et al. A comprehensive proteomic analysis of the type III secretome of Citrobacter rodentium. J Biol Chem 2010;285:6790-6800.

Desvaux M, Hébraud M, Talon R, Henderson IR. Secretion and subcellular localizations of bacterial proteins: a semantic awareness. Trends Microbiol 2009;17:139-45.

Donnenberg MS, Yu J, Kaper JB. A second chromosomal gene necessary for intimate attachment of enteropathogenic Escherichia coli to epithelial cells. J Bacteriol 1993; 175:4670-80.

Egea L, Aguilera L, Giménez R, Sorolla MA, Aguilar J, Badía J, Baldoma L. Role of secreted glyceraldehyde-3-phosphate dehydrogenase in the infection mechanism of enterohemorrhagic and enteropathogenic Escherichia coli: interaction of the extracellular enzyme with human plasminogen and fibrinogen. Int J Biochem \& Cell Biol 2007;39:1190-1203.

Elliot SJ, Hutcheson SW, Dubois MS, Mellies JL, Wainwright LA, Batchelor M et al. Identification of CesT, a chaperone for the type III secretion of Tir in enteropathogenic Escherichia coli. Mol Microbiol 1999;33:1176-89.

Gauthier A, Finlay B. Translocated intimin receptor and its chaperone interact with ATPase of the type III secretion apparatus of enteropathogenic Escherichia coli. J Bacteriol 2003; 185:6747-55.

Houry WA, Frishman D, Eckerskorn C, Lottspeich F, Hartl FU. Identification of in vivo substrates of the chaperonin GroEL. Nature 1999;402:147-54.

Hurmalainen V, Edelman S, Antikainen J, Baumann M, Lähteenmäki K, Korhonen TK. Extracellular proteins of Lactobacillus crispatus enhance activation of human plasminogen. Microbiology 2007;153:1112-22. 
Izoré T, Jov V, Dessen A. Biogenesis, regulation and targeting of the type III secretion system. Structure 2011;19:603-12.

Jarvis KG, Girón JA, Jerse AE, McDaniel TK, Donnenberg MS, Kaper JB. Enteropathogenic Escherichia coli contains a putative type III secretion system necessary for the export of proteins involved in attaching and effacing lesion formation. Proc Natl Acad Sci USA 1995;92:7996-8000.

Jin H, Song YP, Boel G, Kochar J, Pancholi V. Group A streptococcal surface GAPDH, SDH, recognizes uPAR/CD87 as its receptor on the human pharyngeal cell and mediates bacterial adherence to host cells. J Mol Biol 2005;350:27-41.

Kaper JB, Nataro JP, Mobley HL. Pathogenic Escherichia coli. Nat Rev Microbiol 2004; 2:123-40.

Kinoshita H, Uchida H, Kawai Y, Kawasaki T, Wakahara N, Matsuo H et al. Cell surface Lactobacillus plantarum LA 318 glyceraldehyde-3-phosphate dehydrogenase (GAPDH) adheres to human colonic mucin. J Appl Microbiol 2008;104:1667-74.

Laemmli UK. Cleavage of structural proteins during the assembly of the head of bacteriphage T4. Nature1970;222:680-85.

Lama A, Kucknoor A, Mundodi V, Alderete JF. Glyceraldehyde-3-phosphate dehydrogenase is a surface associated, fibronectin-binding protein of Trichomonas vaginalis. Infect Immun 2009;77:2703-11.

Liu CP, Zhou QM, Fan DJ, Zhou JM. PPIase domain of trigger factor acts as auxiliary chaperone site to assist the folding of protein substrates bound to the crevice of trigger factor. Int J Biochem Cell Biol 2010;42:890-901.

Luo W, Donnenberg MS. Analysis of the function of enetropathogenic Escherichia coli EspB by random mutagenesis. Infect Immun 2006;74:810-20. 
Luo W, Donnenberg MS. Interactions and predicted host membrane topology of the enteropathogenic Escherichia coli translocator protein EspB. J Bacteriol 2011;193: 2972-80.

Marlovits TC, Kubori T, Lara-Tejero M, Thomas D, Unger VM, Galan JE. Assembly of the inner rod determines needle length in the type III secretion injectisome. Nature 2006; 441:637-40.

McDaniel TK, Kaper JB. A cloned pathogenicity island from enteropathogenic Escherichia coli confers the attaching and effacing phenotype on K-12 E.coli. Mol Microbiol 1997;23:399-407.

Ochman H, Selander RK. Standard reference strains of Escherichia coli from natural populations. J Bacteriol 1984;157:690-693.

O’Connell CB, Creasey EA, Knutton S, Elliot S, Crowther LJ, Luo W et al. SepL, a protein required for enteropathogenic Escherichia coli type III translocation, interacts with secretion component SepD. Mol Micribiol 2004;52:1613-25.

Page A-L, Parsot C. Chaperones of the type III secretion pathway: jacks of all trades. Mol Microbiol 2002;46:1-11.

Pancholi V, Chhatwal GS. Housekeeping enzymes as virulence factors for pathogens. Int J Med Microbiol 2003;293:391-401.

Parsot C, Hamiaux C, Page A-L. The various and varying roles of specific chaperones in type III secretion systems. Curr Opin Microbiol 2003;6:7-14

Sambrook J, Rusell DW. Molecular cloning: a laboratory manual. Cold Spring Harbor Laboratory, Cold Spring Harbor, NY. 2001. 
Sánchez B, Bressollier P, Urdaci MC. Exported proteins in probiotic bacteria: adhesion to intestinal surfaces, host immunomodulation and molecular cross-talking with the host. FEMS Immunol Med Microbiol 2008;54:1-17.

Sánchez B, Schmitter JM, Urdaci MC. Identification of novel proteins secreted by Lactobacillus rhamnosus GG grown in Mann-Rogosa-Sharpe broth. Lett Appl Microbiol 2009a;48:618-622.

Sánchez B, Schmitter JM, Urdaci MC. Identification of novel proteins secreted by Lactobacillus plantarum that bind to mucin and fibronectin. J Mol Microbiol Biotechnol 2009b;17:158-62.

Schaumburg J, Diekmann O, Hagendorf P, Bergmann S, Rodhe M, Hammerschmidt S et al. The cell wall proteome of Listeria monocytogenes. Proteomics 2004;4:2991-3006.

Seifert KN, McArthur WP, Bleiweis AS, Brady J. Characterization of group B streptococcal glyceraldehyde-3-phosphate dehydrogenase: surface localization, enzymatic activity, and protein-protein interactions. Can J Microbiol 2003;49:350-56.

Sirover MA. New nuclear functions of the glycolytic protein, glyceraldehyde-3-phosphate dehydrogenase, in mammalian cells. J Cell Biochem 2005;95:45-52.

Sirover MA. On the functional diversity of glyceraldehyde-3-phosphate dehydrogenase: biochemical mechanisms and regulatory control. Biochim Biophys Acta 2011;1810: $741-751$.

Stebbins CE, Galán JE. Maintenance of an unfolded polypeptide by a cognate chaperone in bacterial type III secretion. Nature 2001; 414 (6859): 77-81

Stebbins CE, Galán JE. Priming virulence factors for delivery into the host. Nat Rev Mol Cell Biol 2003; 4:738-43. 
TeraoY, Yamaguchi M, Hamada S, Kawabata S. Multifunctional glyceraldehyde-3phosphate dehydrogenase of Streptococcus pyogenes is essential for evasion from neutrophils. J Biol Chem 2006;281:14215-23.

Thomas NA, Deng W, Puente JL, Frey EA, Yip CK, Strynadka NCJ, Finlay BB. CesT is a multi-effector chaperone and recruitment factor required for the efficient type III secretion of both LEE- and non-LEE encoded effectors of enteropathogenic Escherichia coli. Mol Microbiol 2005;57:1762-79.

Thomas NA, Deng W, Baker N, Puente JL, Finlay BB. Hierarchial delivery of an essential host colonization factor in enteropathogenic Escherichia coli. J Biol Chem 2007;282: 29634-45.

Yun M, Park CG, Kim JY, Park HW. Structural analysis of glyceraldehyde 3-phosphate dehydrogenase from Escherichia coli: direct evidence of substrate binding and cofactor-induced conformational changes. Biochemistry 2000;39:10702-10 


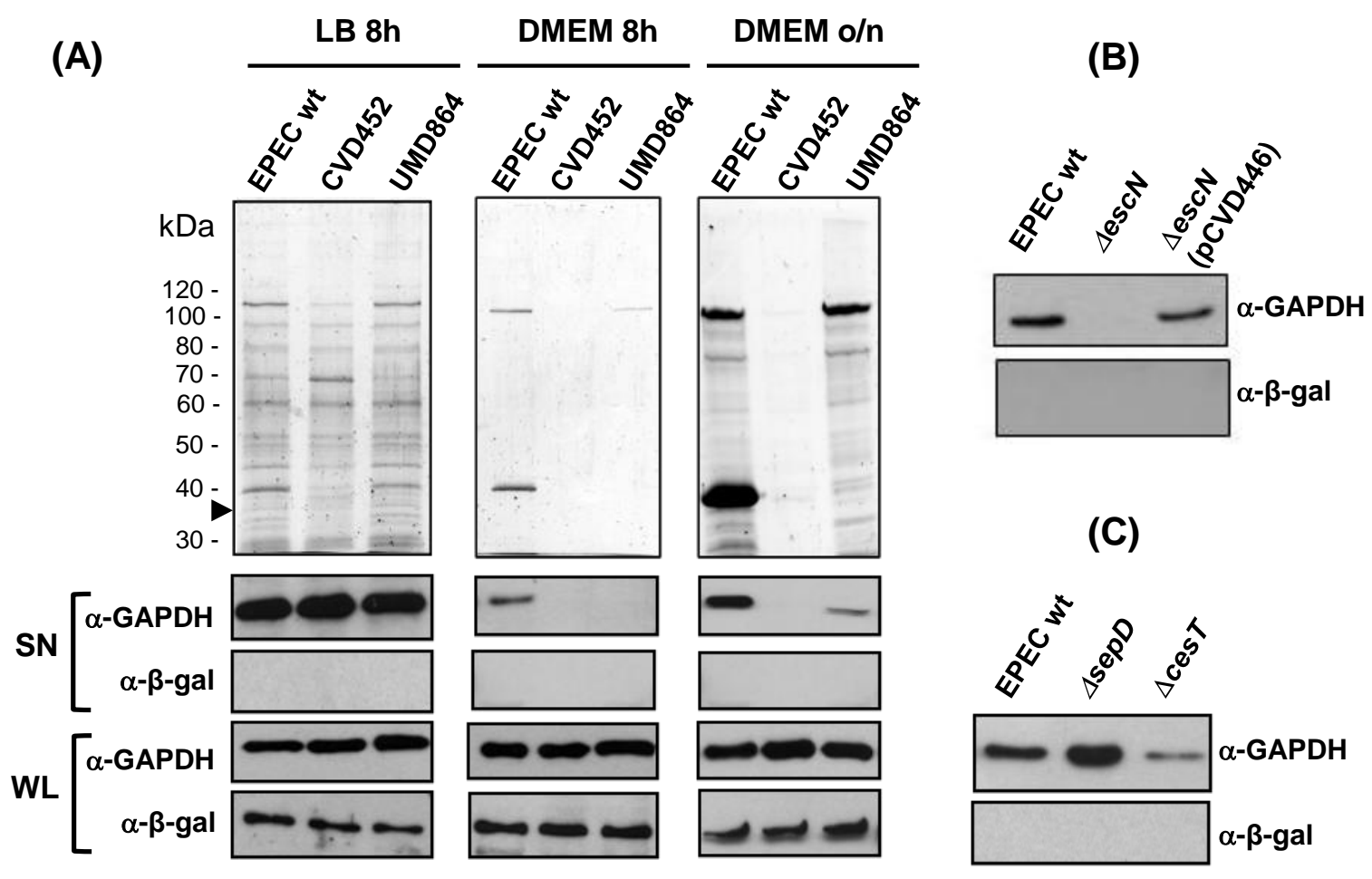




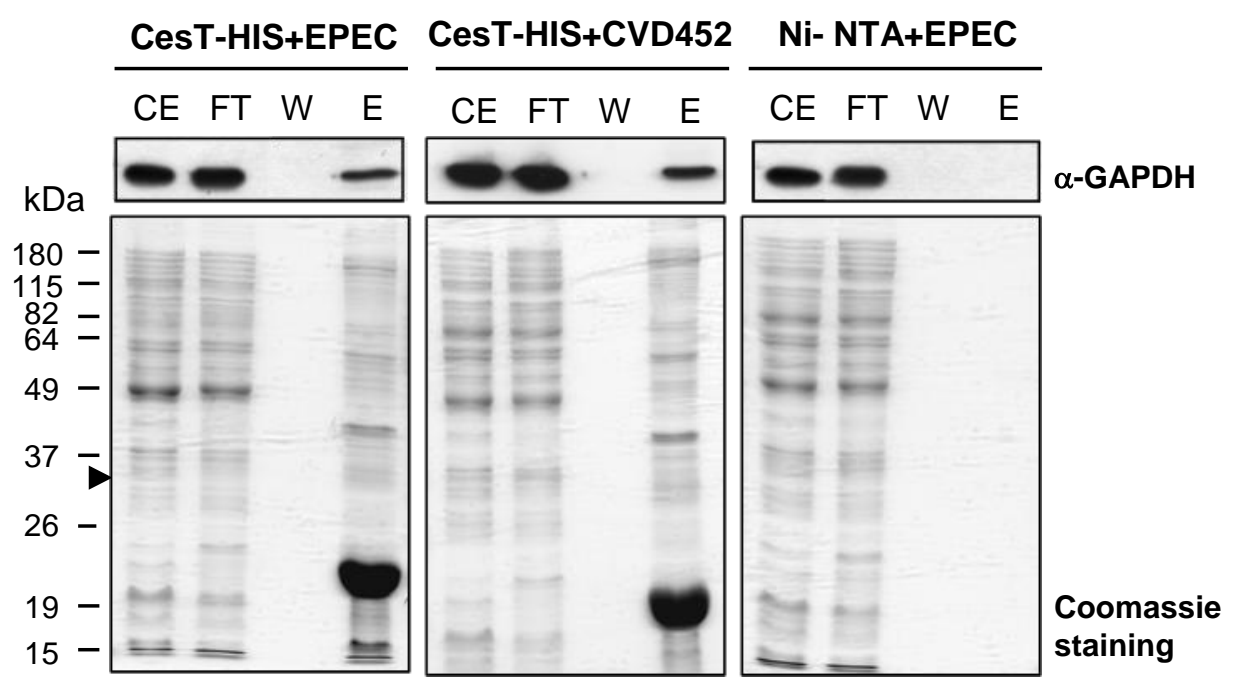




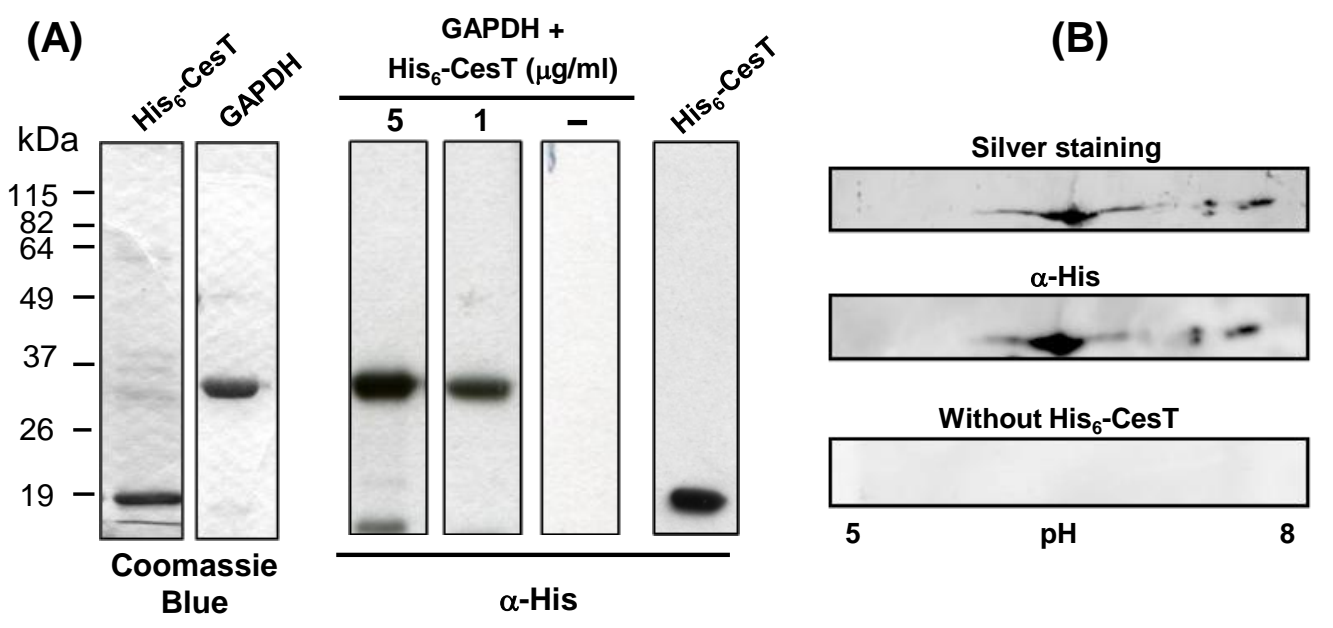


(A)
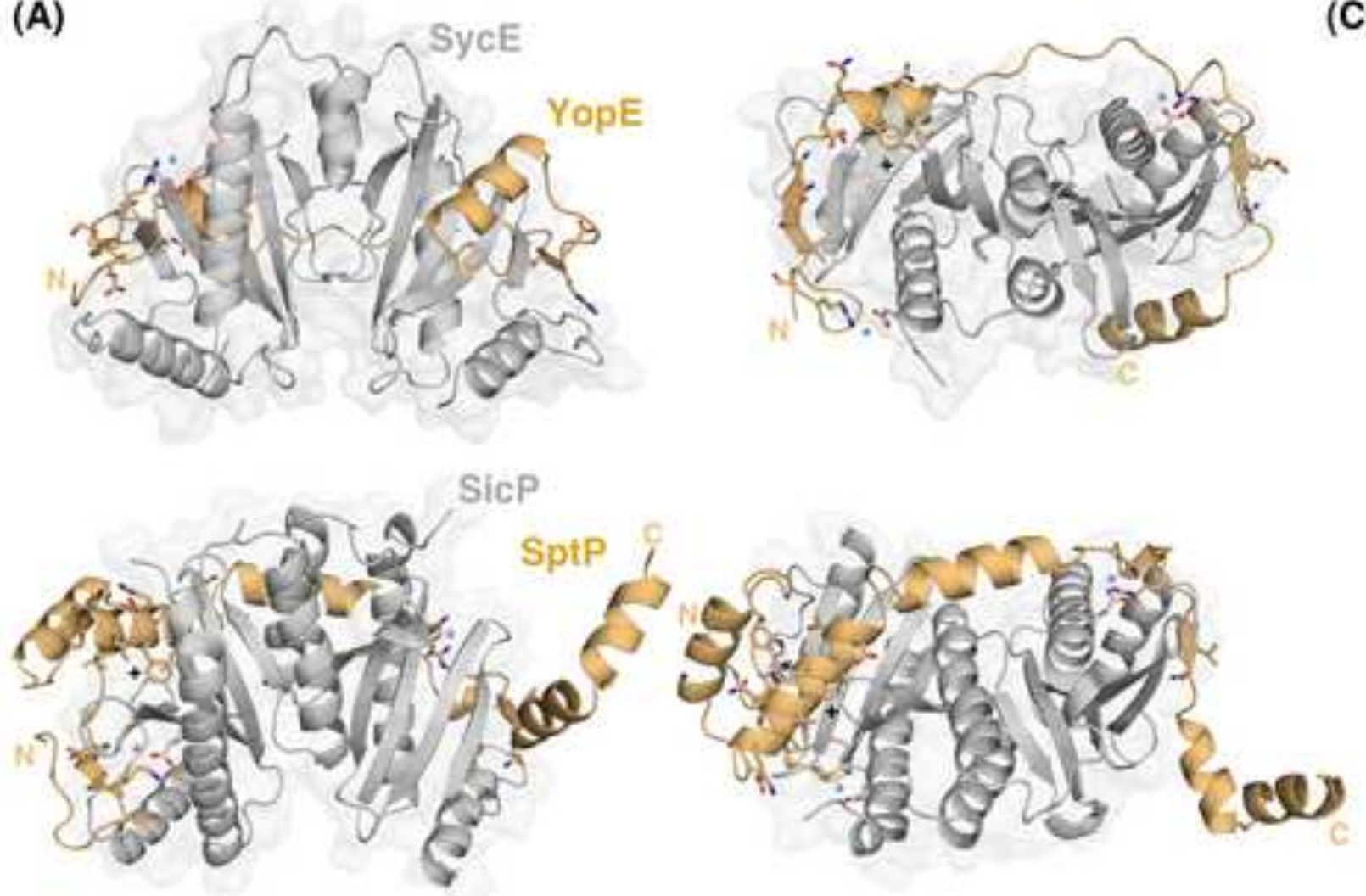

(B)

Yope 28$$
\text { G }
$$

G) TEEEEEE HHHHAHHT STTS EEE

$$
\text { . }
$$

SptP 47 skventedickper TB EEEEE

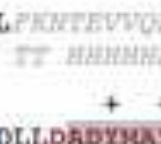

$$
++
$$$$
+
$$

+ +.

LToilaivor

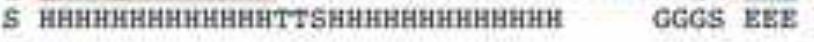
-

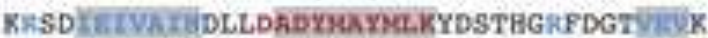
SSEEEEEEESSS HHHHAHHHH TTT S SS EEES

$\operatorname{Gaph} 23$
(C)

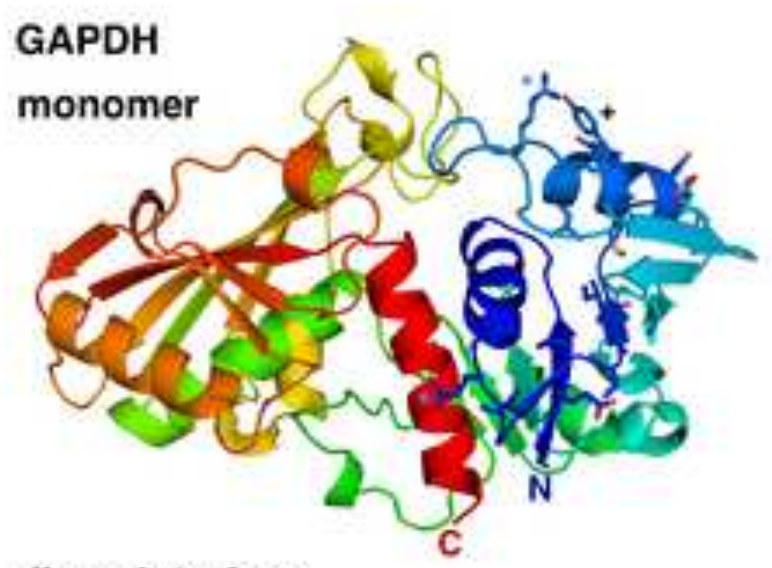

dimer interface

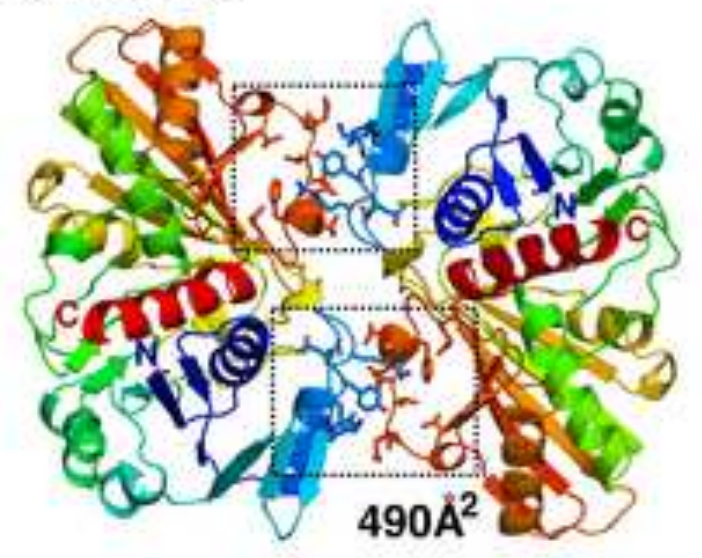




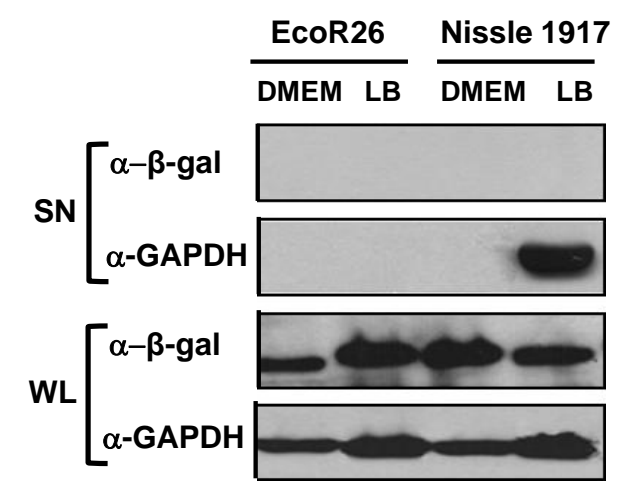




\begin{tabular}{|c|c|c|}
\hline $\begin{array}{l}\text { Strain or } \\
\text { plasmid }\end{array}$ & Genotype or description & Source or reference \\
\hline \multicolumn{3}{|l|}{ E.coli strains } \\
\hline XL1Blue & recAl lac endA1 gyrA96 thi hsdR17 supE44 relAl (F' proAB lacl ${ }^{q}$ lacZ $\left.\triangle \mathrm{M} 15 \mathrm{Tn} 10\right)$ & Stratagene \\
\hline M15 [pREP4] & $\mathrm{F}^{-} \mathrm{Nal}^{\mathrm{s}} \mathrm{Str}^{\mathrm{s}}$ rif $^{\mathrm{s}} \mathrm{lac}^{-} \mathrm{mtl}^{-} \mathrm{recA}^{-}\left[\mathrm{pREP} 4 \mathrm{Kan}^{\mathrm{r}}\right]$ & Qiagen \\
\hline E2348/69 & Wild type EPEC O127:H6 Str ${ }^{r}$ & B.B. Finlay \\
\hline CVD452 & $\mathrm{E} 2348 / 69$ escN::aphA3 & Jarvis et al. (1995) \\
\hline UMD864 & E2348/69 $\Delta$ espB & Donnenberg et al. (1993) \\
\hline$\triangle e s c N$ & Non-polar deletion of $e s c N$ in EPEC E2348/69 & Gauthier and Finlay (2003) \\
\hline$\triangle s e p D$ & Non-polar deletion of sepD in EPEC E2348/69 & Deng et al. (2005) \\
\hline$\Delta c e s T$ & Non-polar deletion of cesT in EPEC E2348/69 & Abe et al (1999) \\
\hline EcoR12 & Human stool isolate & Ochman and Selander (1984) \\
\hline EcoR26 & Human stool isolate & Ochman and Selander (1984) \\
\hline EcoR41 & Human stool isolate & Ochman and Selander (1984) \\
\hline Nissle 1917 & Non-pathogenic probiotic isolate $(\mathrm{O} 6: \mathrm{K} 5: \mathrm{H} 1)$ & Ardeypharm \\
\hline \multicolumn{3}{|l|}{ Plasmids } \\
\hline pCVD446 & BglII DNA fragment of strain E2348/69 containing escN in $\mathrm{pHC} 79, \mathrm{Ap}^{\mathrm{r}}$ & Jarvis et al. (1995) \\
\hline pGex-GapA & pGEX-3x expressing GST-GAPDH, Ap ${ }^{r}$ & Egea et al. (2007) \\
\hline pQE30 & Vector for expression of $\mathrm{N}$-terminal $\mathrm{His}_{6}$-tag proteins, $\mathrm{Ap}^{\mathrm{r}}$ & Qiagen \\
\hline pQE-CesT & pQE30 expressing His $_{6}$-CesT & This study \\
\hline
\end{tabular}

